\title{
Reingeniería del modelado e implementación de simulador de reacciones químicas
}

\section{Reengineering of the modeling and implementation of the chemical reaction simulator}

\author{
MACÍAS-BRAMBILA, Hassem Rubén†*', PULIDO-GONZÁLEZ, Héctor", GÓMEZ-LÓPEZ, \\ Daniela" y ZAMORA-RAMOS, Victor Manuel"
}

'Universidad de Guadalajara - Sistema de Universidad Virtual, Av. Enrique Díaz de León No. 782, Colonia Moderna, C.P. 44190, Guadalajara, Jalisco, México.

"Universidad de Guadalajara - Centro Universitario de Ciencias Exactas e Ingenierías, Blvd. Gral. Marcelino García Barragán No. 1421, Colonia Olímpica, C.P. 44430, Guadalajara, Jalisco, México.

ID $1^{\text {er }}$ Autor: Hassem Rubén, Macías Brambila / ORC ID: 0000-0002-6540-7464, CVU CONACYT ID: 902812

ID $1^{\text {er }}$ Coautor: Héctor, Pulido-González / ORC ID: 0000-0002-8619-3012, CVU CONACYT ID: 313575

ID $2^{\text {do }}$ Coautor: Daniela, Gómez-López / ORC ID: 0000-0002-0748-9211, CVU CONACYT ID: 967451

ID $3^{\text {er }}$ Coautor: Victor Manuel, Zamora-Ramos / ORC ID: 0000-0001-9537-6630, CVU CONACYT ID: 906343

DOI: $10.35429 / J C P E .2020 .23 .7 .9 .18$

Recibido 15 de Julio, 2020; Aceptado 30 de Diciembre, 2020

\section{Resumen}

El presente artículo describe el proceso de análisis, diseño e implementación del proceso de reingeniería para la mejora continua de un sistema distribuido que se integra de una suite de aplicaciones y de los servidores que realizan el procesamiento para la simulación en $2 \mathrm{D}$ y $3 \mathrm{D}$ de reacciones químicas y el reconocimiento de patrones. Este sistema distribuido establece un marco de trabajo para el modelado, representación y simulación de reacciones químicas a través de compuestos y los elementos de la tabla periódica, para el apoyo de las actividades presenciales mediadas por tecnología para las asignaturas de química de las Instituciones de Educación Superior que ofertan estos Programas Educativos. Este proceso de reingeniería se ejecutó con la implementación de la metodología ágil SCRUM para la producción de la suite. Esta suite de aplicaciones permitirá a los estudiantes y profesores a través de las simulaciones la comprensión y entendimiento sobre la interacción de los elementos entre sí, brindando un apoyo en tiempo real, fundamental para la comprensión de tópicos como los electrones de valencia y los enlaces químicos, lo que permitirá sin dudas apoyar el proceso formativo de los estudiantes.

\begin{abstract}
This article describes the process of analysis, design and implementation of the reengineering process for the continuous improvement of a distributed system that is integrated by a suite of applications and servers that perform the processing for the simulation in $2 \mathrm{D}$ and $3 \mathrm{D}$ of chemical reactions and pattern recognition. This distributed system establishes a framework for the modeling, representation and simulation of chemical reactions through compounds and the elements of the periodic table, to support face-to-face activities mediated by technology for the chemistry subjects of the Educational Institutions Superior offered by these Educational Programs. This reengineering process was executed with the implementation of the agile SCRUM methodology for the production of the suite. This suite of applications will allow students and teachers through simulations to understand and understand the interaction of the elements with each other, providing support in real time, fundamental for the understanding of topics such as valence electrons and chemical bonds., which will allow undoubtedly to support the training process of students
\end{abstract}

\footnotetext{
* Correspondencia al Autor (Correo Electrónico: hassem.macias@udgvirtual.udg.mx)

$\dagger$ Investigador contribuyendo como primer autor
} 


\section{Introducción}

Los métodos manuales de resolución de reacciones químicas suelen ser un proceso complicado e incluso puede llegar a ser confuso al tratar con reacciones grandes o que involucran demasiados elementos. Así también, es escaso el número de aplicaciones que hacen simulaciones de reacciones químicas, ya que presentan poca o nula interacción con el usuario.

En la mayoría de los casos se tienen escenarios pre-fabricados o tan solo imágenes, dejando algunos elementos sin contemplar e imposibilitando un acercamiento profundo al fascinante mundo de las reacciones químicas.

Las aplicaciones existentes para generar modelos y/o representaciones de reacciones químicas actualmente son limitadas en cuanto a los tipos de reacciones y a la robustez que implica. Surgiendo así, la necesidad de crear un software con la capacidad de interactuar con los compuestos de manera amigable y directa a nivel molecular para conocer así su estructura, bajo el umbral de la estequiometría. Lo que permite a los usuarios obtener información más detallada de compuestos químicos de una manera más accesible, ordenada y clara. Además de contar con un apoyo visual fundamental para creación de diferentes escenarios donde las reacciones químicas resultan ser trascendentales en objetos de estudio o incluso en la vida cotidiana.

Aprovechando así todas las capacidades que los sistemas de cómputo actual ofrecen, siempre con la intención de crear software rápido, perdurable y estable; y así también adaptar aspectos tales como operatividad y modularidad. Teniendo un producto intuitivo, esto último con el objetivo de que personas que no estén tan versadas en el ámbito de desarrollo puedan manipularlo adecuadamente. $\mathrm{Y}$ así se enfoquen en lo que para ellos realmente importa, la química.

La propuesta desarrollada y que se describe en este artículo comprende los módulos de Arquitectura y Programación de Sistemas en dónde se emplean los algoritmos eficientes en cuanto a tiempo y espacio, capaces de calcular correctamente las reacciones químicas producidas al combinar elementos y compuestos compatibles de la tabla periódica, además de mostrar su simulación.
Así como un uso efectivo de diferentes niveles semánticos, incluyendo la traducción de fórmulas químicas para ser procesadas.

El módulo de Sistemas Distribuidos aplica el uso de hilos de ejecución para el desarrollo de una extensión al módulo principal, con el objetivo de estar siempre a la escucha de archivos para ser procesados y reconocidos.

Aplicando diversos mecanismos $y$ políticas para la coordinación y sincronización de trabajos de cómputo en escenarios de multiprocesador y multicomputador así como el uso y aprovechamiento de sockets de red para la comunicación con la base de datos, y así acceder a los datos necesarios para la simulación y cálculo de las reacciones químicas, además al detalle de los elementos de la tabla periódica.

El módulo Sistemas Inteligentes demuestra la capacidad para construir una solución que simule un fenómeno o sistema químico. Implementando en el sistema, el uso de metodologías de aprendizaje máquina (Machine Learning) con la finalidad de hacer reconocimiento óptico de caracteres (OCR) especializado para compuestos químicos y complementar la accesibilidad del sistema principal.

\section{Metodología}

El desarrollo de este proyecto se llevó a cabo inicialmente en dos periodos semestrales comprendidos en el año 2018, pero a partir del 2019 se realizaron las adecuaciones necesarias a partir de un pilotaje implementado durante el periodo $2019-2020$.

La selección de la metodología para este desarrollo fue SCRUM, ya que desde el inicio del desarrollo fue el método implementado, al permitir la administración de proyectos completos y al estar fundamentada en entregas parciales, logrando así implementaciones con mayor rápidez. En esta metodología se contemplan los roles del equipo Scrum, el experto Scrum y el propietario del proyecto. 
Laínez (2015) define esta metodología como un modelo de desarrollo ideal para las implementaciones incrementales, donde se presentan escenarios complejos y con una alta variación en las especificaciones, funcionalidades o requerimientos. De la misma forma en este modelo se determinaron las fases de análisis, diseño, desarrollo y pruebas.

\section{Análisis}

El inicio del desarrollo se centró en esta primera fase, la cual contempla el desarrollo de un primer entregable denominado pila de producto, documento en el cual se integra información obtenida en la recolección de datos, para su posterior análisis y categorización de la información, lo que permite la definición de las funcionalidades desprendidas de los requerimientos de sistema y usuario, los cuales determinan los requerimientos funcionales y no funcionales del sistema.

El proceso de recolección de datos se ejecutó a través de encuestas sistematizadas usando plataformas especializadas. Se recolectaron datos de cuatro grupos de estudiantes de los Programas Educativos de Técnico Superior Universitario en Química área Tecnología Farmacéutica y Técnico Superior Universitario en Química área Tecnología Ambiental, de la Universidad Tecnológica de Jalisco en su sede Miravalle, derivado de lo anterior y analizando la información recolectada, está fue clasificada y categorizada por funcionalidades, permitiendo así realizar adecuaciones en el documento inicial de Especificación de Requerimientos de Software (ERS) de acuerdo al IEEE 830-1998 del Institute of Electrical and Electronics Engineers (IEEE, 1998); obteniendo así una nueva revisión de los requerimientos funcionales, no funcionales y específicos del sistema, considerando cambios significativos en aspectos de mantenimiento y disponibilidad. A continuación, se muestra un extracto de los requerimientos específicos del ERS:
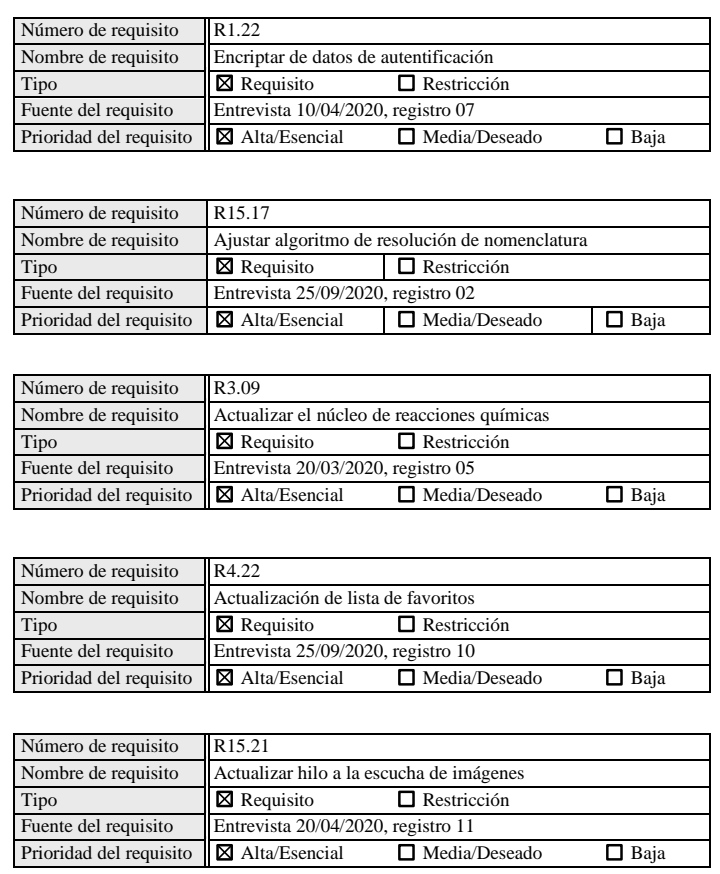

\begin{tabular}{|c|c|c|}
\hline Número de requisito & R5.22 & \\
\hline Nombre de requisito & Filttrar lista de aniones & \\
\hline Tipo & $\square$ Restricción & \\
\hline \begin{tabular}{|l|} 
Fuente del requisito \\
\end{tabular} & Entrevista $12 / 01 / 2020$, registro 05 & \\
\hline Prioridad del requisito & C Media/Deseado & $\square$ Baja \\
\hline
\end{tabular}

Tabla 1 Especificación de requerimientos Fuente: Elaboración propia

$\mathrm{Al}$ término de esta etapa se inició con el desarrollo de la lista de tareas o Sprint Backlog, en el que se determinaron cada una de las actividades a desarrollar, así como la asignación del personal responsable de realizarlas, determinando la duración para cada una de ellas a través de una gráfica de Gantt así como un gráfico de Pert; lo que permite el control en la asignación y planeación de las actividades.

Está lista de tareas brinda los elementos necesarios para realizar pruebas bajo un modelo de desarrollo incremental, además de llevar acabo un modelo de reuniones dónde se lleva a cabo las revisiones de cada uno de los desarrollos, dónde se establecen los acuerdos, ajustes y gestión de riesgos detectados.

\section{Diseño}

Se revisó la arquitectura por capas clienteservidor, simplificando así, la comprensión y organización del desarrollo de sistemas complejos y añadiendo una enorme flexibilidad al diseño de la aplicación, considerando las capas de presentación, la capa de negocio y las capas de datos. A continuación, se muestra un extracto del diagrama de bloques: 


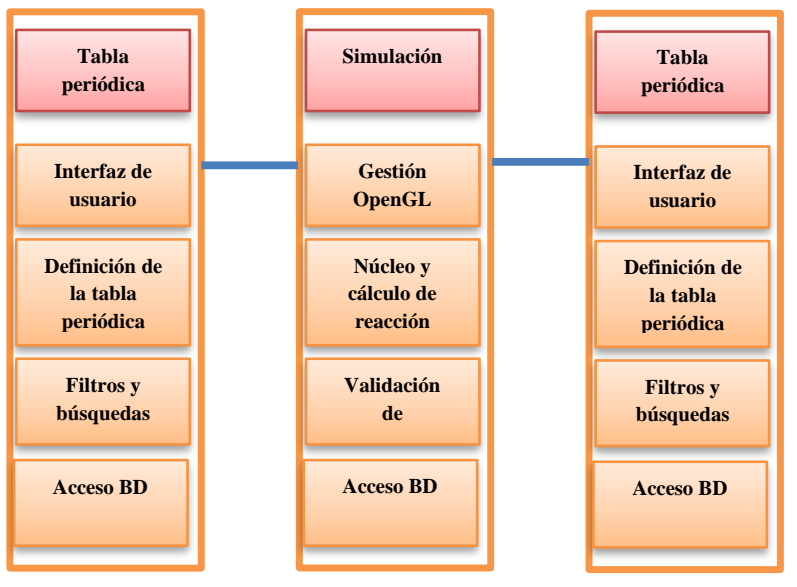

Figura 1 Extracto del diagrama de bloques

Fuente: Elaboración propia

La revisión de los módulos del sistema y su interacción permitió la reestructuración de algunas de las funcionalidades, por lo que a través del Lenguaje Unificado de Modelado (UML), se realizaron las adecuaciones de los componentes estáticos y dinámicos del sistema, iniciando el proceso con el diagrama de clases afectando así las clases que integran el sistema, sus atributos, métodos y las dependencias o relaciones entre ellas.

Esta tarea impactó en la revisión de las tarjetas Clase-Responsabilidad-Relación (CRC), para su análisis con mayor detalle con respecto a las dependencias existentes entre cada una de las clases, a continuación, se presenta una tarjeta CRC de elementos:

\begin{tabular}{|c|c|}
\hline \multicolumn{2}{|c|}{$\begin{array}{c}\text { Elemento } \\
\text { Superclase: tabla }\end{array}$} \\
\hline Atributos & Métodos \\
\hline $\begin{array}{l}\text {-codigo: integer (4) } \\
\text { - nombre: } \operatorname{varchar}(40) \\
\text { - categoria: } \operatorname{varchar}(40)\end{array}$ & $\begin{array}{l}\text { + Clasificacion(): void } \\
+ \\
\text { setNombre(varchar[]): } \\
\text { void getNombre(): } \\
+\quad \text { varchar[] } \\
+ \\
\text { setCategoria(varchar[] } \\
\text { ): void } \\
+ \text { getCategoria(): } \\
\text { varchar[] }\end{array}$ \\
\hline Responsabilidades & Colaboraciones \\
\hline Clasificación con grupos & Grupos \\
\hline $\begin{array}{ll}\text { Clasificación } & \text { de } \\
\text { categorías } & \end{array}$ & Categorías \\
\hline
\end{tabular}

Tabla 2 Tarjeta CRC categoría Fuente: Elaboración propia
Un elemento más que se revisó fue el diagrama de objetos, el cual se actualizó con el objetivo de mantener una eficiente comunicación e identificación de entradas y salidas entre los objetos del sistema que tienen relaciones activas entre ellas y que comparten mensajes, los cuales fueron modificados en su estructura y periodicidad por las modificaciones realizadas desde el diagrama de clases. La correcta validación de este proceso de comunicación asegura que los datos de entrada y salida alimenten cada uno de los procesos que dependen de ellos.

Los casos de uso del sistema fueron actualizados de tal manera que desde esta representación gráfica se verificó la interacción y las condiciones que se requieren para su ejecución, considerando en todo momento los supuestos y restricciones de cada uno de los requerimientos que integran las funcionalidades del sistema. A continuación, se muestra el caso de uso de reacciones:

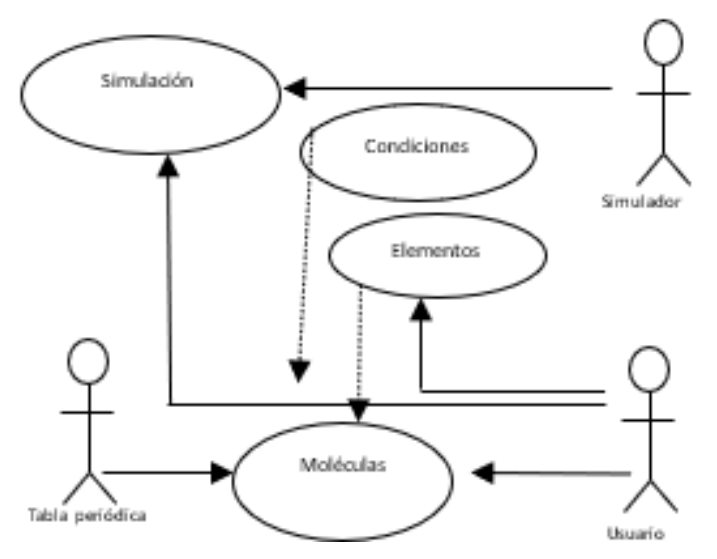

Figura 2 Diagrama de casos de uso reacciones Fuente: Elaboración propia

La restructuración en el modelado de los casos de uso permitió la redefinición de los mismos, especificando los procesos de cada uno de los actores, así como las actividades del sistema y sus condicionantes o alternativas. A continuación, se muestra la definición del caso de uso agregar elemento: 


\begin{tabular}{|c|c|c|c|}
\hline \# & $\begin{array}{l}\text { Actividad } \\
\text { de actor }\end{array}$ & $\begin{array}{l}\text { Actividad de sistema } \\
\text { [Descripción/Cálculo] }\end{array}$ & $\begin{array}{l}\text { [Condición] } \\
\text { Alternativa }\end{array}$ \\
\hline 1 & $\begin{array}{l}\text { Presionar } \\
\text { el botón } \\
\text { de agregar } \\
\text { elemento }\end{array}$ & $\begin{array}{l}\text { El sistema despliega la } \\
\text { vista de la creación de } \\
\text { elementos, solicitando } \\
\text { los datos a llenar }\end{array}$ & $\begin{array}{l}\text { - Si la conexión } \\
\text { a internet } \\
\text { falla, la vista } \\
\text { no puede } \\
\text { cargarse y se } \\
\text { envía un } \\
\text { mensaje de } \\
\text { error }\end{array}$ \\
\hline 2 & $\begin{array}{l}\text { Ingresar } \\
\text { datos }\end{array}$ & $\begin{array}{l}\text { El sistema validará la } \\
\text { información y la } \\
\text { almacenará en la base } \\
\text { de datos }\end{array}$ & $\begin{array}{l}\text { - Si no existen } \\
\text { evaluaciones } \\
\text { registradas } \\
\text { aparecerá en } \\
\text { blanco el } \\
\text { control. } \\
\text { - Si la conexión } \\
\text { con la base de } \\
\text { datos falla, el } \\
\text { sistema no } \\
\text { almacenará la } \\
\text { información y } \\
\text { enviará un } \\
\text { mensaje de } \\
\text { error. } \\
\text { - Si un campo } \\
\text { queda vacío } \\
\text { no se podrá } \\
\text { guardar y se } \\
\text { enviará un } \\
\text { mensaje de } \\
\text { error. }\end{array}$ \\
\hline 3 & & $\begin{array}{l}\text { El sistema guardará un } \\
\text { registro en otra tabla } \\
\text { con el identificador del } \\
\text { elemento. }\end{array}$ & \\
\hline
\end{tabular}

Tabla 3 Definición de caso de uso agregar elemento Fuente: Elaboración propia

La adecuación de la definición de los casos de uso facilitó implementar las modificaciones identificadas en las actividades de altas, bajas y modificaciones de los mensajes, así como la definición de las nuevas entradas y salidas de cada uno de los procesos.

De la misma manera se realizó una revisión y adecuación del diseño semántico de datos implementados en el sistema, lo que incluyó la revisión y adecuación del modelo conceptual del sistema a través del diagrama entidad-relación (DER) identificando las relaciones vigentes y su pertinencia, así como las restricciones funcionales $y$ dependencias determinadas por estas relaciones. A continuación, se presenta un extracto del DER:

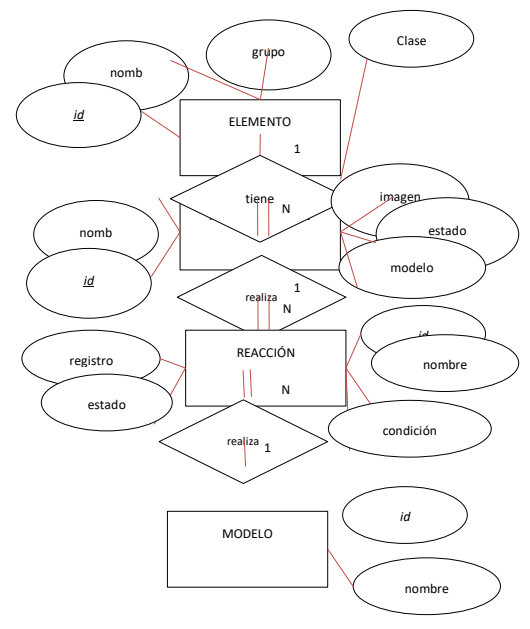

Figura 3 Extracto del DER

Fuente: Elaboración propia

Este proceso de revisión y actualización del modelo conceptual obliga a la revisión y modificación del proceso derivado del modelo relacional, verificando en todo momento que se lleve a cabo el proceso de normalización de la base de datos y su respectiva verificación de restricciones. A continuación, se muestra un extracto del modelo relacional:

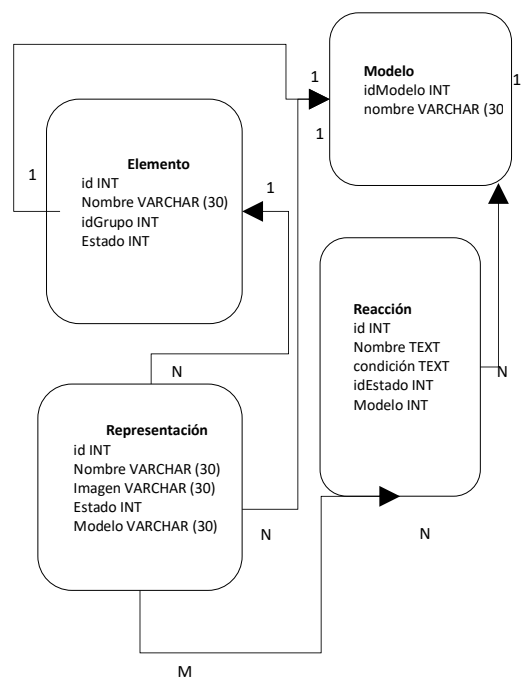

Figura 4 Extracto del modelo relacional Fuente: Elaboración propia

\section{Desarrollo}

La fase de desarrollo de software se llevó a cabo después de contar con la actualización de los requerimientos y de los modelados correspondientes, se inició de acuerdo al modelo de capas definidas desde la primera entrega y liberación del sistema, facilitando el control de versiones y actualizaciones de las mejoras implementadas. A continuación, se muestra la interfaz del reconocimiento de compuestos, es dependiente del código; mismo que se vinculará con la aplicación de escritorio en el módulo correspondiente:

MACÍAS-BRAMBILA, Hassem Rubén, PULIDOGONZÁLEZ, Héctor, GÓMEZ-LÓPEZ, Daniela y ZAMORARAMOS, Victor Manuel. Reingeniería del modelado e implementación de simulador de reacciones químicas. Revista de Energía Química y Física. 2020 


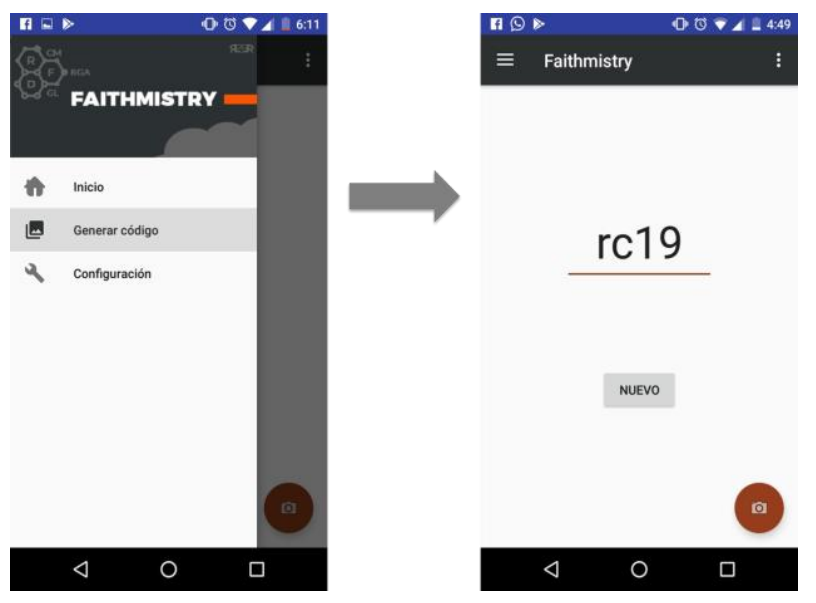

Figura 5 Interfaz de reconocimiento de compuestos Fuente: Elaboración propia

El sistema brinda la opción de ver los detalles de cada elemento presionando su número atómico en la tabla. Esto muestra la información que conforma cada elemento de una manera más ordenada y fácil; estas propiedades son indispensables para el correcto funcionamiento de los módulos principales. A continuación, se muestra la interfaz de propiedades:

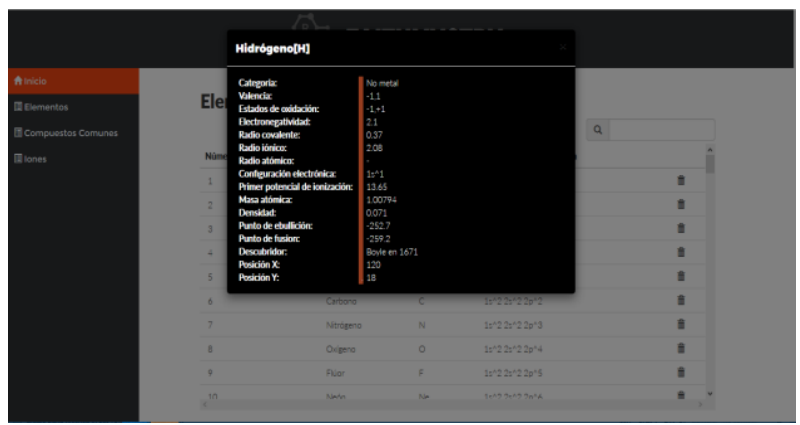

Figura 6 Interfaz de reconocimiento de compuestos Fuente: Elaboración propia

En el módulo dónde las valencias y el arreglo de átomos son enviados al núcleo de simulación en el cual, dependiendo de la elección del usuario, serán tratados para realizar una síntesis o una descomposición. A continuación, se muestra la interfaz del algoritmo de simulación:

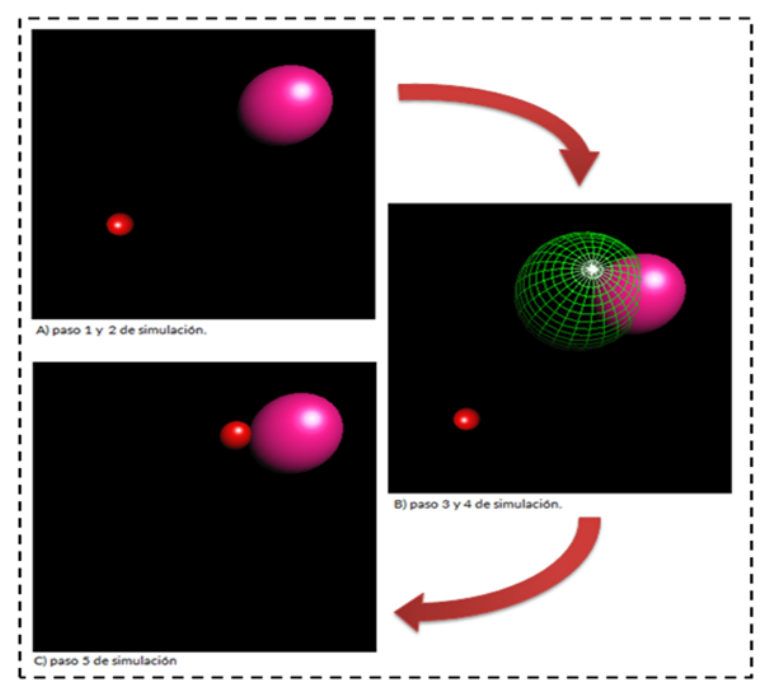

Figura 7 Interfaz de simulación

Fuente: Elaboración propia

Para lo anterior se estableció el siguiente algoritmo:

Paso 1: seleccionar los dos primeros elementos del arreglo y posicionar sus átomos aleatoriamente.

Paso 2: asignarles sus respectivas valencias y calcular la carga del producto.

Paso 3: usando las reglas descritas en la teoría de repulsión de pares de electrones de valencia, aplicar la configuración para el producto correspondiente a su carga y la proporción de los elementos en él.

Paso 4: asignar los puntos de atracción al elemento que servirá de centro y distribuir la carga del elemento entre cada uno de los puntos de atracción.

Paso 5: calcular las colisiones de cada uno de los elementos que formarán el compuesto en relación con los puntos de atracción del átomo central y reubicar los elementos colisionados.

Paso 6: meter los elementos con carga residual, de nuevo en la lista.

Paso 7: repetir desde el paso 1 hasta que no existan más elementos. 
El cálculo de colisiones es una adaptación de la distancia euclidiana para un espacio. El algoritmo marca los átomos que contienen puntos de unión como "no colisionables", calcula una esfera por cada punto de atracción y se incrementa su radio hasta entrar en contacto con un átomo "colisionable", el átomo alcanzado es marcado como "no colisionable", la esfera de colisiones es eliminada y el elemento colisionado es movido gradualmente hasta el centro del punto de atracción. A continuación, se muestra una interfaz de la representación de las colisiones:

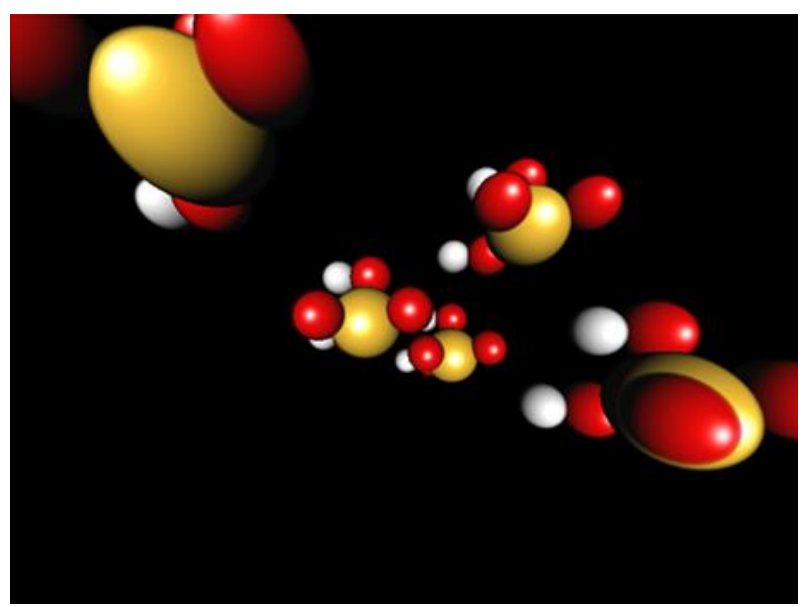

Figura 8 Representación final de colisiones Fuente: Elaboración propia

Para controlar e interactuar directamente con las simulaciones de las reacciones químicas se cuenta con una serie de eventos vinculados al teclado los cuales son:

\begin{tabular}{|c|c|}
\hline Tecla & Acción \\
\hline 1 & $\begin{array}{l}\text { Activar/Desactivar efecto de Blur en } \\
\text { simulación }\end{array}$ \\
\hline 2 & $\begin{array}{l}\text { Activar/Desactivar efecto de átomos } \\
\text { sólidos en simulación }\end{array}$ \\
\hline 3 & $\begin{array}{l}\text { Activar/Desactivar esfera de } \\
\text { colisiones }\end{array}$ \\
\hline $\mathbf{R}$ & $\begin{array}{l}\text { Disminuir la resolución de la } \\
\text { simulación }\end{array}$ \\
\hline $\mathbf{T}$ & $\begin{array}{l}\text { Aumentar la resolución de la } \\
\text { simulación }\end{array}$ \\
\hline- & Disminuir el zoom de la cámara \\
\hline+ & Aumentar el zoom de la cámara \\
\hline Espacio & $\begin{array}{l}\text { Comenzar la simulación de la } \\
\text { fórmula seleccionada }\end{array}$ \\
\hline
\end{tabular}

Tabla 4 Control del simulador con teclado Fuente: Elaboración propia

Este control de las simulaciones opera el proceso, a continuación, se muestra la simulación con efecto sólido:
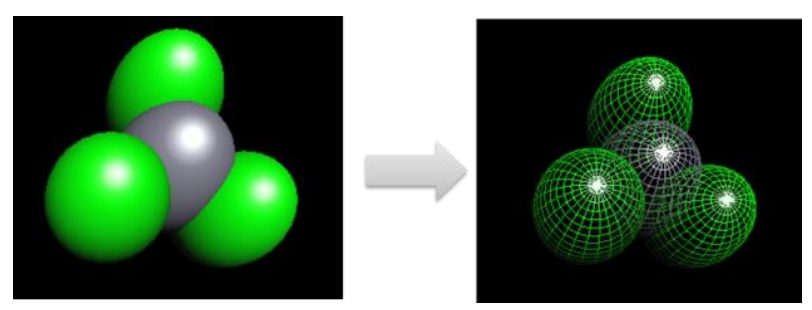

Figura 9 Representación final de colisiones Fuente: Elaboración propia

De la misma forma operan los controles para la simulación con esferas de colisiones, tal como se muestra a continuación:
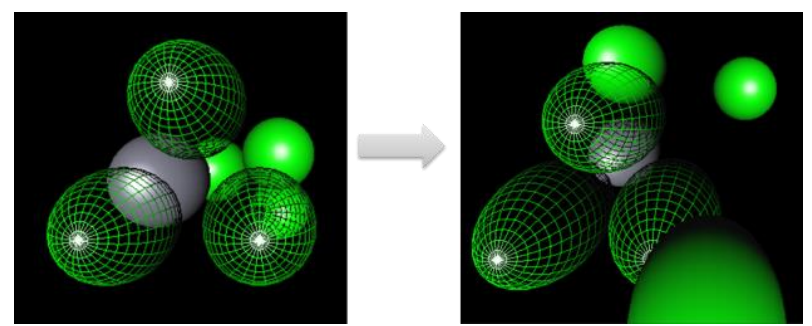

Figura 10 Simulación con esferas de colisiones Fuente: Elaboración propia

Por último, se muestra el proceso de simulación con extremos de resolución:
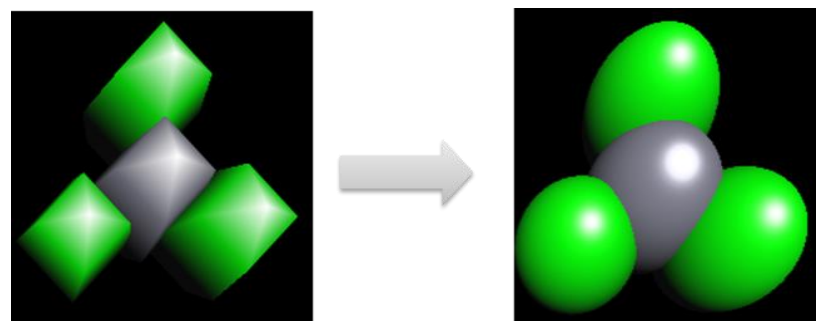

Figura 11 Simulación con extremos de resolución Fuente: Elaboración propia

\section{Pruebas}

En esta fase se recuperó el plan de pruebas original desarrollado en la primera versión del software y a este se incluyeron aspectos de configuración, procesamiento $\mathrm{y}$ actualización de los procesos. En este nuevo documento se integraron elementos del historial de versiones, así como el nuevo alcance de las pruebas: elementos nuevos a probar, funcionalidades a probar, pruebas de regresión, determinación de las funcionalidades a no probar y las estrategias de pruebas. La valoración del desempeño esperado de realizó a través de la actualización de la definición de casos de uso y de los modelos actualizados para los aspectos arquitectónicos y semánticos de datos, los criterios de aceptación y rechazo de los hallazgos de las pruebas se determinó a través de un comportamiento binario.

MACÍAS-BRAMBILA, Hassem Rubén, PULIDOGONZÁLEZ, Héctor, GÓMEZ-LÓPEZ, Daniela y ZAMORARAMOS, Victor Manuel. Reingeniería del modelado e implementación de simulador de reacciones químicas. Revista de Energía Química y Física. 2020 


\section{Resultados}

Se actualizaron los componentes del sistema distribuido en su primera versión, a través del mismo modelo cliente-servidor, verificando la correcta colaboración entre la suite de aplicaciones que realizan las peticiones al servidor de datos y de procesamiento, asegurando que los dispositivos móviles no requieran realizar procesamientos de la detección de patrones.

Derivado de la baja complejidad del almacenamiento de datos se continua con un modelo centralizado para su administración, derivado de la estimación realizada a la capacidad operativa de una jornada en los laboratorios de química de los Programas Educativos de Técnico Superior Universitario en Química área Tecnología Ambiental, Técnico Superior Universitario en Química Área Tecnología Farmacéutica, Ingeniería en Tecnología Ambiental e Ingeniería en Química Farmacéutica, ya que no se tiene una conectividad simultánea de más de 50 usuarios, por lo que las peticiones de los clientes (modelo cliente-servidor), la gestión del propio almacenamiento y de las operaciones, justifica continuar con el esquema centralizado.

La determinación del entorno de desarrollo, instalación y configuración del servidor se mantiene bajo el supuesto de software libre, por lo que no se requiere la adquisición de algún tipo de licenciamiento, lo que facilita y favorece la adaptación de las tecnologías sin costos de licenciamiento.

En el plan de pruebas se consideraron aspectos de procesamiento de datos, peticiones y resolución de las mismas, disponibilidad de conectividad, rendimiento y experiencia de usuario; se comprobó la conexión simultánea de 141 usuarios y la atención a las respectivas peticiones, así como el procesamiento y validación de las salidas de los procesos, con estadística descriptiva donde se determina en un $97.05 \%$ con los resultados esperados en los casos de prueba. Para el resto de salidas y pruebas que no cumplieron los criterios de aceptación se documentaron y se reprocesaron.

\section{Conclusiones}

La simulación de reacciones químicas para el proceso de aprendizaje de los Programas Educativos son fundamentales para el aseguramiento del proceso de desarrollo de las capacidades declaradas en los planes y programas de estudio, además de brindar un acceso de herramientas tecnológicas, que incluso en situaciones sanitarias como la que en este momento obligó la suspensión de actividades presenciales, permitió brindar continuidad a las secuencias de aprendizaje de las asignaturas de química.

Este proceso de mejora continua en el funcionamiento y rendimiento del sistema obliga a continuar con acciones y estrategias concretas que permitan fortalecer y consolidar el sistema distribuido, actualizándolo y haciéndolo cada vez más eficiente.

Un reto importante es la adecuación de estas funcionalidades móviles a un entorno de iOS, ya que actualmente el acceso solo se cuenta para la plataforma Android, situación que permitirá iniciar una nueva fase e incrementar el alcance de este sistema a todos los escenarios posibles.

\section{Referencias}

Aguiar, B. O., Velázquez, R. M., \& Aguiar, J. L. (2019). Innovación docente y empleo de las TIC en la Educación Superior. Revista ESPACIOS, 40(02).

Chang, R. (2017). Química General. (7 Ed). McGraw-Hill.

Corey, R. B., \& Pauling, L. (1953). Molecular models of amino acids, peptides, and proteins. Review of Scientific Instruments, 24(8), 621627.

D. Hearn, M.P. Baker (2011). Gráficos por computadora con OpenGL, 3a edición. Pearson Prentice Hall.

Date, C.J. (2001). Introducción a los sistemas de bases de datos. Pearson Education. 251-253.

Dimes, Troy. (2015). Conceptos básicos de Scrum: desarrollo de software agile y manejo de proyectos agile. Babelcube. 9-14. 
Martínez Mora, O. (2015). Química inorgánica. Gillespie R., Hargittai I., (2012) The VSEPR model of molecular geometry Ed. Dover.

Hargittai, I., \& Chamberland, B. (1986). The VSEPR model of molecular geometry. In Symmetry (pp. 1021-1038).

Hernández, F. G., Granados, A. P., \& Gallegos, S. R. (2016). Química I. Santillana. IUPAC. Compendium of Chemical

Hernández, Giovanni; Martínez, Álvaro; Jiménez, Robinson \& Jiménez, Franklin. (2019). Scrum y peopbleware: elementos clave para la gestión en la construcción de software. Risti. 265-277.

IEEE. (1998). IEEE Std 830-1998 - IEEE Recommended Practice for Software Requirements Specifications. mayo 20, 2017, de C - IEEE Computer Society Sitio web: https://standards.ieee.org/findstds/standard/8301998.html.

Izquierdo, J. F., Cunil, F., Tejero, J., Iborra, M., \& Fité, C. (2004). Cinética de las Reacciones Químicas. Edicions de la Universitat de Barcelona.

J.D. Foley, A. van Dam, S.K. Feiner, J.F. Hughes (1996). Computer graphics: principles and practice, 2 nd edition in C. Addison-Wesley.

Kenneth Connors (1990). Chemical Kinetics, VCH Publishers.

Kernighan B.W, Ritchie M.D. (2015). The C Programing Lenguage. (2 ed.) Pearson.

Koltun, W. L. (1965). U.S. Patent No. 3,170,246. Washington, DC: U.S. Patent and Trademark Office.

Kotz, J. C., Treichel, P. M., \& Townsend, J. R. (2009). Chemistry \& Chemical Reactivity. Thomson Books /Cole.

Laínez Fuentes José Rubén (2015) Desarrollo de Software Ágil Extremme Programming y Scrum (2nd edition) Ed. IT Campus Academy.

Leigh, G. J. (Ed.). (1990). Nomenclature of inorganic chemistry: recommendations 1990. Oxford: Blackwell scientific publications.
León, A., Daniel, J., \& Tabares Hurtado, J. D. (2019). Desarrollo de un simulador de control automático de procesos en Microsoft Excel para ser utilizado en el laboratorio virtual de control y simulación de procesos.

Morales, J. P., Hurtado, J. I., Mejía, A. T., \& Cuartas, J. H. (2019). Metodología de Ajuste de un NMPC con Sistema de Inferencia Borrosa Takagi Sugeno y Conjuntos Borrosos Multidimensionales para Aplicaciones en Procesos Químicos no Lineales. Revista Iberoamericana de Automática e Informática industrial, 16(1), 100-113.

Morris Hein y Arena Susan (2014). Foundations of collage chemistry (14 ed.). John Wiley \& Sons Inc.

Muñoz, J. C. M. (2014) Aplicación de una estrategia didáctica que permita la comprensión de la estequiometría a partir de un aprendizaje significativo. Universidad Nacional de Colombia.

Parry Robert (1987). Chemistry, Experimental Foundations, Prentice-Hall, Inc.

Pilone, D., Pitman, N. (2009). UML 2.0 in a Nutshell. O'Reilly Media, Inc.

Ríos, A. (2019). Implementación de un modelo hidrodinámico tridimensional en el embalse de Paso Severino. Aportes para la modelación de calidad de agua.

Romero J. Especificación de Requisitos Software según el estándar IEEE 830. Recuperado el día 8 de agosto de 2017 de: https://www.fdi.ucm.es/profesor/gmendez/docs/ is0809/ieee830.pdf

Russell, S., \& Norving, P. (2004). Inteligencia Artificial: Un enfoque moderno. Pearson. San Fabián, J. Aguado, A. y de Ema, I.,

Scott, F. H. (2008). Elementos de Ingeniería de las Reacciones Químicas. (cuarta, Ed.) Mexico: Person Educación.

Sommerville, I. (2015). Software Engineering (10 ed.). Person.

Terrazas Pastor, Rafael (2011). Planificación y programación de operaciones. Scielo. 8-11. 
Tuya, Javier; Ramos Roman, I.\& Dolado Cosín, J. (2007). Técnicas cuantitativas para la gestión en la ingeniería de software. Netbiblio. 49-53.

MACÍAS-BRAMBILA, Hassem Rubén, PULIDOGONZÁLEZ, Héctor, GÓMEZ-LÓPEZ, Daniela y ZAMORARAMOS, Victor Manuel. Reingeniería del modelado e implementación de simulador de reacciones químicas. Revista de Energía Química y Física. 2020 